\title{
MATROIDS DETERMINE THE EMBEDDABILITY OF GRAPHS IN SURFACES
}

\author{
THOMAS ZASLAVSKY
}

(Communicated by Thomas Brylawski)

\begin{abstract}
The embeddability of a graph in a given surface is determined entirely by the polygon matroid of the graph. That is also true for cellular embeddability in nonorientable surfaces but not in orientable surfaces.
\end{abstract}

An embedding of a finite graph $\Gamma$ in a surface $S$ is a homeomorphism of $\Gamma$, regarded as a topological space, with a closed subset of $S$. In order to know in which surfaces $\Gamma$ embeds it suffices to consider only the compact surfaces: the orientable ones $T_{g}$ of genus $g$ (Euler characteristic 2-2g) for $g \geq 0$, and the nonorientable ones $U_{h}$ of Euler characteristic $2-h$ for $h \geq 1$. For uniformity of terminology we define the demigenus $d$ of a compact surface by $d\left(T_{g}\right)=2 g$, $d\left(U_{h}\right)=h$. One knows exactly which compact surfaces can embed $\Gamma$ if one knows two parameters: the genus of the graph, $g(\Gamma)=\min \{g: \Gamma$ embeds in $\left.T_{g}\right\}$, and its crosscap number (also called nonorientable genus) $h(\Gamma)=\min \{h: \Gamma$ embeds in $\left.U_{h}\right\}$. A natural companion to these is the demigenus of $\Gamma$ (also known as generalized genus, Euler genus, etc.),

$$
d(\Gamma)=\min \{2 g(\Gamma), h(\Gamma)\},
$$

the smallest demigenus of a compact surface in which $\Gamma$ embeds. It is the purpose of this note to point out the apparently unrecognized fact that these three parameters are matroidal, that is, determined by the polygon matroid ${ }^{1}$ of the graph. This fact, which generalizes Whitney's theorem that planarity is matroidally determined [20], follows readily from published work on graph embedding. $^{2}$

Received by the editors October 17, 1988 and, in revised form, January 25, 1989.

1980 Mathematics Subject Classification (1985 Revision). Primary 05C10; Secondary 05B35.

Key words and phrases. Primary: graph embedding, genus, demigenus, crosscap number, genus range, crosscap range; Secondary: polygon matroid, cycle matroid.

Research supported by various grants, notably DMS-8606102 and DMS-8808239 of the National Science Foundation.

${ }^{1}$ Also called the 'cycle matroid'. Its circuits are the circuits of the graph. Cf. $[17, \S 1.10]$ or $[18, \S 6.1]$.

${ }^{2}$ A general reference for graph embedding is [8]. For matroids see [17] or [18, 19]. 
Three operations on a graph $\Gamma$ are (a) identifying two vertices in different components, (b) the reverse, and (c) twisting. The last named consists in splitting $\Gamma$ into subgraphs $\Gamma_{1}$ and $\Gamma_{2}$ whose intersection is precisely a 2-separating vertex set $\{v, w\}$ such that $v$ and $w$ are connected by a path in $\Gamma_{1}$ and $\Gamma_{2}$, and reconnecting all the edges of $\Gamma_{2}$ at $v$ and $w$ to the opposite vertex, respectively $w$ or $v$. Plainly, none of these operations changes the polygon matroid $G(\Gamma)$. Whitney's 2-isomorphism theorem $[21 ; 17, \S 6.1 ; 18, \S 6.3]$ states that, if $G(\Gamma)=G\left(\Gamma^{\prime}\right)$, then $\Gamma^{\prime}$ can be obtained from $\Gamma$ by iterating operations $(\mathrm{a}, \mathrm{b}, \mathrm{c})$. Thus we need to show that these operations do not alter the genus, demigenus, or crosscap number.

The BHKY theorem [2], that $g(\Gamma)=\sum_{1}^{n} g\left(\Gamma_{i}\right)$ where $\Gamma_{1}, \ldots, \Gamma_{n}$ are the blocks of $\Gamma$, shows that genus is unaffected by (a) and (b). The observation of [15, Corollary 2] that the analogous formula holds for the demigenus of a connected graph implies a similar conclusion for $d(\Gamma)$ if $\Gamma$ is connected. A simple argument from [15] gives the crosscap number as well: For any graph let $\delta(\Gamma)=h(\Gamma)-d(\Gamma)$. As noted in [15, Eq. (1)], $\delta(\Gamma)=0$ or 1 . Now let $\Gamma$ have blocks $\Gamma_{1}, \ldots, \Gamma_{n}$. If $\Gamma$ is connected,

$$
h(\Gamma)=d(\Gamma)+\delta(\Gamma)=\sum_{i=1}^{n} d\left(\Gamma_{i}\right)+\delta(\Gamma) .
$$

[15, Theorem 1] states that

$$
\delta(\Gamma)=1 \quad \Leftrightarrow \quad \text { all } \delta\left(\Gamma_{i}\right)=1 .
$$

Thus $h(\Gamma)$ is determined by the blocks of $\Gamma$.

If $\Gamma$ has $k>1$ components we use a trick of $\left[2\right.$, p. 567]. Let $\Gamma^{\prime}$ be $\Gamma$ with $k-1$ edges added to make a connected graph. In $\Gamma^{\top}$ the blocks are $\Gamma_{1}, \ldots, \Gamma_{n}$ and single edges $\Gamma_{n+1}, \ldots, \Gamma_{n+k-1}$. The latter have genus and demigenus 0 and $h\left(\Gamma_{i}\right)=\delta\left(\Gamma_{i}\right)=1$. Since the genus, demigenus, and crosscap number of $\Gamma^{\prime}$ are independent of the location of the extra edges, it is easy to see that $g\left(\Gamma^{\prime}\right)=g(\Gamma)$ and $h\left(\Gamma^{\prime}\right)=h(\Gamma)$, whence $d\left(\Gamma^{\prime}\right)=d(\Gamma)$ and $\delta\left(\Gamma^{\prime}\right)=\delta(\Gamma)$. It follows that $d(\Gamma)=\sum_{1}^{n} d\left(\Gamma_{i}\right)$ and that (1) and (2) hold for $\Gamma$. Therefore $h(\Gamma)$ is determined by the blocks of $\Gamma$.

Now suppose $\Gamma=\Gamma_{1} \cup \Gamma_{2}$ where $\Gamma_{1}$ and $\Gamma_{2}$ are connected ${ }^{3}$ and $\Gamma_{1} \cap \Gamma_{2}$ consists of just the two vertices $v$ and $w$. The main theorem of Decker et al. [3; 4, Theorem 0.1$]$ is that there is a function $\mu(\Gamma,\{v, w\})$ of connected graphs with a distinguished vertex pair such that

$$
g(\Gamma)=g\left(\Gamma_{1}\right)+g\left(\Gamma_{2}\right)+\left\lceil\frac{1}{4}\left(3-\mu\left(\Gamma_{1},\{v, w\}\right) \mu\left(\Gamma_{2},\{v, w\}\right)\right)\right\rceil .
$$

If $\Delta$ is a graph and $v, w \in V(\Delta)$, let $\Delta^{v w}$ be $\Delta$ with an extra edge $v w$ adjoined. Richter [10] proves that

$$
d(\Gamma)=\min \left\{d\left(\Gamma_{1}^{v w}\right)+d\left(\Gamma_{2}^{v w}\right), d\left(\Gamma_{1}\right)+d\left(\Gamma_{2}\right)+2\right\} .
$$

\footnotetext{
${ }^{3}$ The requirement of connectedness is not stated in [4] but it is necessary for the proof. The formula may be false if $\Gamma_{1}$ or $\Gamma_{2}$ does not contain a $v w$ path.
} 
In [9] he shows by a more complicated argument that there is a function $\mu$ of pairs of connected graphs with a distinguished vertex pair (this $\mu$ is unrelated to that of Decker et al.) such that

$$
h(\Gamma)=h\left(\Gamma_{1}\right)+h\left(\Gamma_{2}\right)+\mu\left(\left(\Gamma_{1},\{v, w\}\right),\left(\Gamma_{2},\{v, w\}\right)\right) .
$$

These three formulas, together with additivity on blocks, imply that $g(\Gamma)$, $d(\Gamma)$, and $h(\Gamma)$ are invariant under twisting. Hence our main result:

Theorem. The genus, demigenus, and crosscap number of a graph are determined by its polygon matroid.

By a minor of a graph or matroid $B$ we mean any isomorph of a contraction of a subgraph or submatroid of $B$. The relation defined by $A \leq B$ if $A$ is a minor of $B$ is a partial ordering of isomorphism types of graphs and also of matroids; we call it the minor ordering. It is easy to see that for each surface $S$ the property of embeddability in $S$ is hereditary, that is, if $\Gamma$ embeds so does every minor. Consequently there is a set $\mathscr{F}_{G}(S)$ of graphs (actually, isomorphism types of graphs) such that $\Gamma$ is embeddable in $S$ if and only if no minor of $\Gamma$ belongs to $\mathscr{F}_{G}(S)$. The members of $\mathscr{F}_{G}(S)$ are known as the forbidden graph minors for embedding in $S$. Our theorem implies:

Corollary 1. A graph $\Gamma$ embeds in $S$ if and only if $G(\Gamma)$ has no minor in the set $\mathscr{F}_{M}(S)=\left\{G(F): F \in \mathscr{F}_{G}(S)\right\}$.

Corollary 2. A matroid $M$ is the matroid of a graph embeddable in $S$ if and only if it is graphic and has no minor belonging to $\mathscr{F}_{M}(S)$.

In other words, the class of matroids whose graphs are embeddable in a given surface is determined by forbidden matroid minors (since the property of graphicity is so determined, according to the famous theorem of Tutte [16]; the five forbidden minors are described in $[17, \S 10.5]$ and $[19, \S 2.6]$ ). By [12] (see also [11]), and when $S=U_{h}$ also by [1], $\mathscr{F}_{G}(S)$ is finite. Consequently the forbidden minors for a matroid to be the polygon matroid of an $S$-embeddable graph are finite in number.

One might hope that $\mathscr{F}_{M}(S)$ would be much smaller than $\mathscr{F}_{G}(S)$, which is very large if $d(S) \geq 2$. But this is not the case for $S=T_{0}$ or $U_{1}$, as one can see by inspection of the two forbidden graph minors for $T_{0}$ (i.e., $K_{5}$ and $K_{3,3}$ ) and the 35 for $U_{1}$ (they are the first 35 irreducible graphs listed in [7]).

Corollaries 1 and 2 remain true if $S$ is replaced by a pair of surfaces $T_{g}$ and $U_{h}$ and embeddability is interpreted as being embeddable in both, or in either, of the surfaces. In either case the forbidden graph minors are finitely many, by [13] and in the second case by [1], hence so are the forbidden matroid minors.

A natural follow-up queston is whether cellular embeddability of a graph in a given surface, where every component of the graph's complement in $S$ is an open 2-cell, is a matroidal property. To avoid triviality assume $\Gamma$ is a connected graph. The genus range is $\mathfrak{g}(\Gamma)=\{g: \Gamma$ has a cellular embedding in $\left.T_{g}\right\}$ and the crosscap range is $\mathfrak{h}(\Gamma)=\left\{h: \Gamma\right.$ embeds cellularly in $\left.U_{h}\right\}$. Each 
of these sets is finite and, if nonempty, is contiguous: if $i<k<j$ and $i, j$ are in the set, so is $k$. (See [5, Theorem 3.2] and [14, Theorem 8], or consult $[8, \S 3.4]$.) Obviously $g(\Gamma)=\min \mathfrak{g}(\Gamma)$; we define $g_{\max }(\Gamma)=\max \mathfrak{g}(\Gamma)$. It is clear that $\mathfrak{h}(\Gamma)=\varnothing$ if $\Gamma$ is a tree and otherwise $h(\Gamma)=\min \mathfrak{h}(\Gamma)$; we set $h_{\max }(\Gamma)=\max \mathfrak{h}(\Gamma)$. From work of Edmonds [6] it follows directly (see [8, Theorem 3.4.3]) that $h_{\max }(\Gamma)=\beta_{1}(\Gamma)$, the cyclomatic number of $\Gamma$. This is precisely the nullity of $G(\Gamma)$. Thus the crosscap range is matroidal.

A theorem of Xuong ([22]; see [8, Corollary to Theorem 3.4.13]) says that $g_{\max }(\Gamma)=\frac{1}{2}\left(\beta_{1}(\Gamma)-\xi(\Gamma)\right)$, where $\xi(\Gamma)$ is the minimum over all spanning trees $T$ of the number of components of $\Gamma \backslash E(T)$ which have an odd number of edges. This quantity is unfortunately not determined by $G(\Gamma)$. For example let $\Gamma_{1}$ and $\Gamma_{2}$ be formed from the three blocks $K_{3}, K_{3}, K_{2}$. To construct $\Gamma_{1}$ we join each $K_{3}$ to a different vertex of the $K_{2}$. Obviously $\xi\left(\Gamma_{1}\right)=2$. To form $\Gamma_{2}$ we join the two $K_{3}$ 's at a vertex and attach the $K_{2}$ anywhere. Evidently $\xi\left(\Gamma_{2}\right)=0$. Yet the two graphs have the same matroid because they have the same blocks.

To summarize:

Proposition. The crosscap range of a connected graph is determined by its matroid, but the genus range is not.

One wonders how much information about the genus range is lost by passing to the matroid. Let $g_{\max }(M)$, for a graphic matroid $M$, be

$$
\max \left\{g_{\max }(\Gamma): G(\Gamma)=M\right\} .
$$

Is $g_{\max }(M)-g_{\max }(\Gamma)$ for graphs with $G(\Gamma)=M$ bounded by a constant, or by a small multiple of $g_{\max }(M)$ ?

\section{REFERENCES}

1. D. Archdeacon and P. Huneke, A Kuratowski theorem for nonorientable surfaces, J. Combin. Theory Ser. B 46 (1989), 173-231.

2. J. Battle, F. Harary, Y. Kodama, and J. W. T. Youngs, Additivity of the genus of a graph, Bull. Amer. Math. Soc. 68 (1962), 569-571. MR 27 \#5247.

3. R. W. Decker, The genus of certain graphs, Ph.D. dissertation, Ohio State University, 1978.

4. R. W. Dēcker, H. H. Glover, and J. P. Huneke, Computing the genus of the 2-amalgamations of graphs, Combinatorica 5 (1985), 271-282. MR 87f:05054.

5. R. A Duke, The genus, regional number, and Betti number of a graph, Canad. J. Math. 18 (1966), 817-822. MR 33 \#4917.

6. J. Edmonds, On the surface duality of linear graphs, J. Res. Nat. Bur. Standards (U.S.A.) Sect. B 69B (1965), 121-123. MR 32 \#444.

7. H. H. Glover, J. P. Huneke, and C. S. Wang, 103 graphs that are irreducible for the projective plane, J. Combin. Theory Ser. B 27 (1979), 332-370. MR 81h:05060.

8. J. L. Gross and T. W. Tucker, Topological graph theory, Wiley-Interscience, New York, 1987.

9. B. Richter, On the non-orientable genus of a 2-connected graph, J. Combin. Theory Ser. B 43 (1987), 48-59.

10. __ On the Euler genus of a 2-connected graph, J. Combin. Theory Ser. B 43 (1987), 60-69. 
11. N. Robertson and P. D. Seymour, Generalizing Kuratowski's theorem, in Proc. Fifteenth Southeastern Conf. on Combinatorics, Graph Theory and Computing (Baton Rouge, 1984), Congressus Numerantium 45 (1984), 129-138. MR 86f:05058.

12. __, Graph minors: VIII. A Kuratowski theorem for general surfaces, submitted.

13. __ Graph minors : $X V$. Wagner's conjecture, submitted.

14. S. Stahl, Generalized embedding schemes, J. Graph Theory 2 (1978), 41-52. MR 58 \#5318.

15. S. Stahl and L. W. Beineke, Blocks and the nonorientable genus of graphs, J. Graph Theory 1 (1977), 75-78. MR 57 \#161.

16. W. T. Tutte, Lectures on matroids, J. Res. Nat. Bur. Standards (U.S.A.) Sect. B 69B (1965), 1-47. MR 31 \#4023. Reprinted with commentary in D. McCarthy and R. G. Stanton, eds., Selected papers of W. T. Tutte, vol. II, Charles Babbage Research Centre, St. Pierre, Man., Canada, 1979, 439-496.

17. D. J. A. Welsh, Matroid theory, Academic Press, London, 1976. MR 55 \#148.

18. Neil White, ed., Theory of matroids, Encycl. of Math. and Its Appl., vol. 26, Cambridge Univ. Press, Cambridge, Eng., 1986. MR 87k:05054.

19. _ Combinatorial geometries, Encycl. of Math. and Its Appl., vol. 29, Cambridge Univ. Press, Cambridge, Eng., 1987. MR 88g:05048.

20. H. Whitney, Non-separable and planar graphs, Trans. Amer. Math. Soc. 34 (1932), 339-362.

21. _ _ 2-isomorphic graphs, Amer. J. Math. 55 (1933), 245-254.

22. N. H. Xuong, How to determine the maximum genus of a graph, J. Combin. Theory Ser. B 26 (1979), 217-225. MR 80k:05051.

Department of Mathematics, State University of New York, University Center at Binghamton, Binghamton, New YoRK 13901 